\title{
ON A MAXIMAL IDEAL SPACE SEPARATED BY A PEAK POINT
}

\author{
JOSEPH E. SOMMESE
}

ABstract. The purpose of this note is to answer in the negative the following question raised by Gamelin [1]: if $A$ is a function algebra which has the property that $X$, the spectrum of $A$, is expressible as the union of two compact sets $X_{1}$ and $X_{2}$ which have as their intersection a peak point $p$ of $X$, and if $f \in C(\mathrm{X})$ satisfies $\left.\left.f\right|_{x_{1}} \in A\right|_{x_{1}}$ and $\left.\left.f\right|_{x_{2}} \in A\right|_{x_{2}}$, then is $f \in A$ ? The counterexample is obtained by the use of a construction which is applicable to general function algebras. Let $A$ be a function algebra and $I$ a proper closed ideal, denoting by $A[I]$ the set $\{(f, f+s): f \in A$, $s \in I\}$, it is shown that $A[I]$ is a function algebra which has as its spectrum two copies of the spectrum of $A$ identified along hull (I).

Let $A$ be a function algebra with spectrum $X$ and $I$ a proper closed ideal of $A$, then $A[I]$ will denote the set $\{(f, f+s): f \in A, s \in I\}$. It is readily verified that $A[I]$ is a uniformly closed subalgebra of $A \oplus A$ which contains the constants.

The functions in $A \oplus A$ can be regarded as continuous functions on two disjoint copies of $X$, so that $A[I]$ can be regarded as an algebra of continuous functions on the space $X \#^{I} X$ obtained from the disjoint union of two copies of $X$ by identifying each point in the first copy belonging to the hull of $I$ to the corresponding point in the second copy.

Denote by $\Sigma(A[I])$ the spectrum of $A[I]$.

Lemma. $\Sigma(A[I])=X \#^{I} X$.

Proof. If $x \in$ hull $(I)$ define $\epsilon_{x}: A[I] \rightarrow C$ by $\epsilon_{x}(f, f+s)=f(x)$ and if $x \notin$ hull $(I)$ define $\boldsymbol{\epsilon}_{x}^{j}: A[I] \rightarrow C$ for $j=1,2$ by $\boldsymbol{\epsilon}_{x}^{1}(f, f+s)=f(x)$, $\epsilon_{x}^{2}(f, f+s)=f(x)+s(x)$. As $x$ runs through $X$ these homomorphisms run through the points in $X \#^{I} X$.

The embedding $f \rightarrow(f, f)$ of $A$ in to $A[I]$ determines the dual projection $\lambda \rightarrow \lambda^{*}$ of $\Sigma(A[I])$ into $X$ defined by $\lambda^{*}(f)=\lambda(f, f), f \in A$. This projection collapses $X \#^{I} X$ onto $X$.

Case I. $\lambda^{*} \in$ hull $(I)$.

For all $t \in I, \lambda(t, t)=\lambda^{*}(t)=0$ and since $\lambda(t, 0)+\lambda(0, t)=\lambda(t, t)=0$, it follows that $\lambda(t, 0)=-\lambda(0, t)$. Since $\lambda(t, 0) \cdot \lambda(0, t)=0, \lambda$ annihilates $\{0\} \oplus I$ and $I \oplus\{0\}$. If $\lambda_{1} \in \Sigma(A[I])$ and $\lambda_{1}^{*}=\lambda^{*}$, then $\lambda_{1}-\lambda$ annihilates $\{0\} \oplus I, I \oplus\{0\}$ and the embedded copy of $A$ which was

Received by the editors February 20, 1970.

AMS 1969 subject classifications. Primary 4655.

Key words and phrases. Function algebra, maximal ideal space, peak point. 
$\{(f, f): f \in A\}$. Thus $\lambda_{1}=\lambda$ and $\lambda^{*}$ has precisely one preimage in $\Sigma(A[I])$.

Case II. $\lambda^{*} \notin \operatorname{hull}(I)$.

For all $t \in I$ define $\phi_{1}(t)=\lambda(t, 0)$ and $\phi_{2}(t)=\lambda(0, t)$. One of $\phi_{1}, \phi_{2}$ is identically zero, for if not then there exist $s, t \in I$ with $\phi_{1}(s) \neq 0$, $\phi_{2}(t) \neq 0$ but $\phi_{1}(s) \phi_{2}(t)=\lambda(s, 0) \lambda(0, t)=0$, a contradiction. When both $\phi_{1}$ and $\phi_{2}$ are identically zero, $\lambda^{*} \in \operatorname{hull}(I)$ and the proof reduces to the previous case. Accordingly suppose $\phi_{2}$ vanishes identically, then $\lambda(f, f+s)=\lambda^{*}(f)+\phi_{2}(f)=\lambda^{*}(f)$; if $\phi_{1}$ vanishes identically $\lambda(f, f+s)=\lambda(f+s-s, f+s)=\lambda^{*}(f+s)-\phi_{1}(s)=\lambda^{*}(f+s)$. Thus, if $\phi_{1}$ vanishes identically $\lambda=\epsilon_{\lambda^{*}}^{2}$, and if $\phi_{2}$ vanishes identically $\lambda=\epsilon_{\lambda^{*}}^{1}$. This finishes the proof of the lemma.

Let $A$ denote the disc algebra, i.e. the algebra of all continuous complex-valued functions on the unit circle of the complex plane whose negative Fourier coefficients vanish. The closed ideals of this algebra have been studied in some detail. From this theory we will need the fact [2] or [3] that if $J$ is the closed ideal generated by the function

$$
g(z)=(z-1) \exp ((z+1) /(z-1))
$$

then $J$ is contained in precisely one maximal ideal, namely, the one topologically generated by $z-1$. Moreover, this inclusion is proper.

To produce the counterexample, form the function algebra $A[J]$ which has the space obtained by identifying two copies of the closed unit disc at the point 1 as its spectrum. Denote the two closed discs by $\bar{\Delta}_{1}$ and $\bar{\Delta}_{2}$. These discs intersect on the point 1 which is a peak point for the function $((z+1) / 2,(z+1) / 2)$ in $A[J]$.

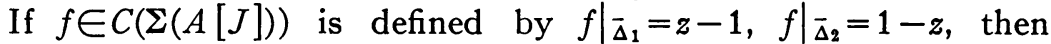
$f \notin A[J]$, for if so, then the functions $z-1$ and $1-z$ differ by an element of $J$ whence $2(z-1) \in J$. This conclusion contradicts the quoted result about $J$, so the counterexample is complete.

I wish to thank Professor Stuart J. Sidney for several conversations and suggestions which were helpful to me in the preparation of this note.

\section{BiBLIOGRAPHY}

1. T. W. Gamelin, Embedding Riemann surfaces in maximal ideal spaces, J. Functional Analysis 2(1968), 123-146. MR 36 \#6941.

2. K. Hoffman, Banach spaces of analytic functions, Prentice-Hall Series in Modern Analysis, Prentice-Hall, Englewood Cliffs, N. J., 1962. MR 24 \#A2844.

3. W. Rudin, The closed ideals in an algebra of analytic functions, Canad. J. Math. 9(1957), 426-434. MR 19, 641.

Yale University, New Haven, Connecticut 06520 